\title{
European Science Open Forum-ESOF2006 München, 15-18 July, 2006. Report on the session. Green chemistry: a tool for socio- economic development and environmental protection
}

DOI: $10.1039 / \mathrm{b} 611593 \mathrm{~b}$

\begin{abstract}
Green chemistry claims success at the 2006 European Science Open Forum. The session "Green chemistry: a tool for socio-economic development and environmental protection" was a popular one as is described by Pietro Tundo, Alvise Perosa and Alberto Procopio.
\end{abstract}

ESOF2006 was the second pan-European General Science Meeting held in Munich at the Forum am Deutschen Museum and the Deutsches Museum from July 15th to 19th, 2006, whose purpose was to promote interaction and dialogue between science and the general public.

Seven scientific themes were chosen to reflect the sessions: Combining Cultures, Dealing with Disasters, Earth and Environment, Particles and Planets, Science and Society, Fabric of Science, Workings of Life.

Finally success for green chemistry! It claimed its place on the same level as other more popular scientific disciplines and topical issues such as natural disaster prevention, biodiversity, genomics, evolution, medical science, and astronomy.

Within the Earth and Environment theme, green chemistry was indicated as one of the main options for environmental safeguarding, and this was highlighted on the posters placed at the entrance of the meeting. Plus, the session on green chemistry that took place in Munich represented the only session on chemistry.

"Is green chemistry a real option?" This question shows clearly what the press and society ask of chemistry. And a positive answer to this question was given by the panellists: Tundo, Poliakoff, Seddon, Mours, and Sydnes during the $2.5 \mathrm{~h}$ long session held on Sunday afternoon entitled: "Green chemistry: a tool for socio-economic development and environmental protection".

The crowded event was moderated by Barbara Schwerdtfeger, a science

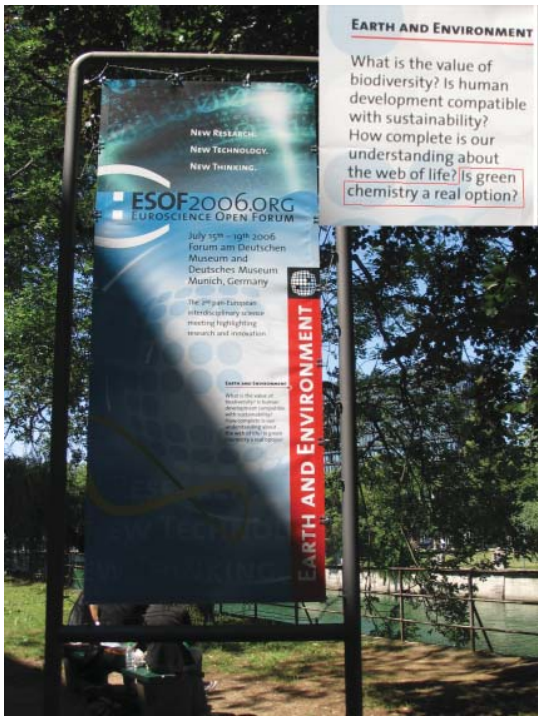

journalist. Among the participants were Francesco Fedi president of COST (European Cooperation in the field of Science and Technical Research), and Howard Moore senior advisor of ICSU (International Council for Science), and of the press (Berliner Zeitung), all wanted to know why green chemistry is an option, and why they should care.

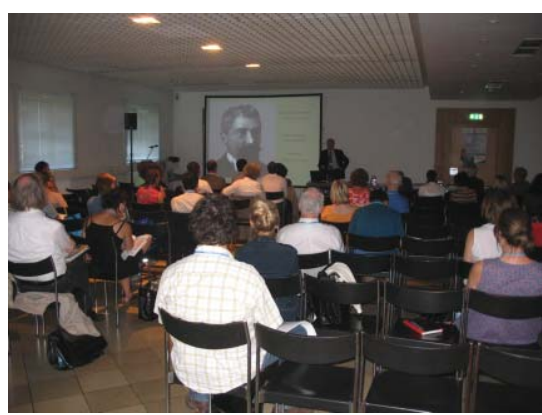

The definition of green chemistry was illustrated by Martyn Poliakoff from Nottingham, who also showed how green chemistry is starting to move from academic research to industrial applications.

The international context, expectations, and philosophy were described in depth by Pietro Tundo from Venice, who placed emphasis on the need of green chemistry to foster development in developing nations, and on the newest international networking initiatives (see Box 1 and Box 2). Kenneth Seddon from Belfast illustrated one of the most recent and important networks, the International Green Network, founded by the G8 ministers of research, which is now starting thanks to national government funding of its activities.

Marian Mours of CEFIC (European Chemical Industry Council) offered the

IGN The mission of the International Green Network (IGN) includes research, coordination and sponsorship for scientific collaborations, targeted training for a new generation of scientists, and the support of sustainable development. IGN consists of eight research centres, one in each of the G8 countries, and it will accelerate movement towards a sustainable energy and materials economy by bringing together scientists, engineers, research institutions, firms, analysts and government regulators. 
MEGREC The Mediterranean Green Chemistry Network constitutes a platform for the development of research and training in green chemistry in the countries of the Mediterranean basin, with focus on water management, exploitation of local natural resources, production and use of fertilizers, and monitoring and reducing the presence of toxic compounds in the food chain. With a clear focus on local priorities, but with the extended know-how of all the partners.

industrial perspective on green chemistry, and Leiv Sydnes, past president of IUPAC, from Bergen demonstrated how green chemistry is needed in order to address some of the major concerns affecting humanity, such as poverty, lack of resources in the developing nations, and widespread pollution.

The presentations were followed by an hour long discussion based on questions

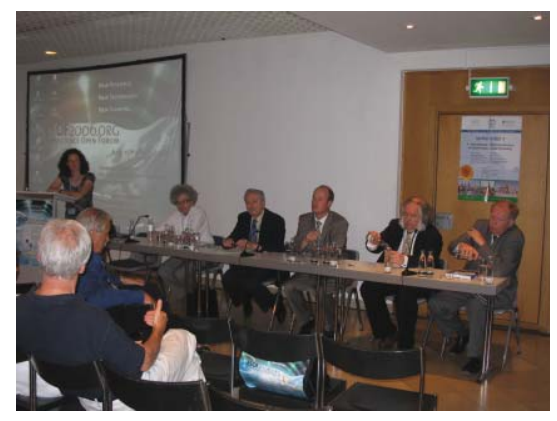

from the audience. Both science policy and research issues were addressed, as well as possible actions on how to improve the public understanding of chemistry.

Later on the same day, an informal and entertaining "round-up" of the day's sessions was presented by Quentin Cooper, a BBC radio science journalist. During this informal, and widely attended event two of the five speakers represented the green chemistry session: Ken Seddon and Martyn Poliakoff. They were targeted with numerous questions by the audience and proved that the green chemistry session was a large success and that awareness for chemistry is certainly rising.

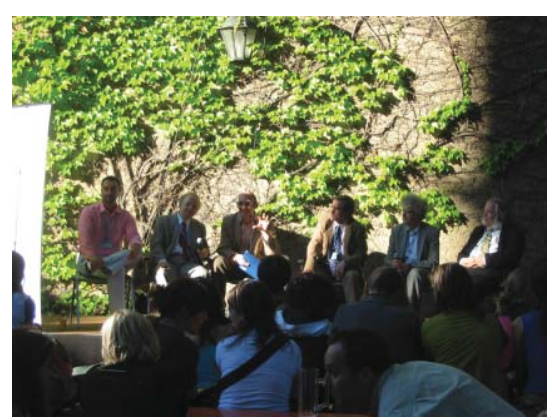

The idea of this session was initiated, proposed and supported by the Interuniversity Consortium, INCA (www.unive.it/inca). It is the latest of a series of initiatives that are placing Europe in the lead in green chemistry.

Pietro Tundo, Alvise Perosa and Alberto Procopio

Consorzio Interuniversitario Nazionale "la Chimica per l'Ambiente" INCA, Italy 